\title{
New method of accounting for interference contributions within a multiperipheral model
}

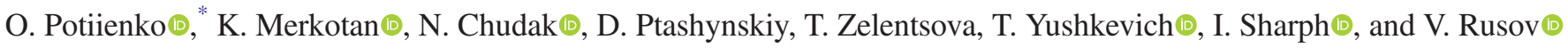 \\ Odessa National Polytechnic University, Schevchenko avenue 1, Odessa 65044, Ukraine
}

(Received 15 January 2020; accepted 1 April 2020; published 24 April 2020)

\begin{abstract}
We consider an inelastic scattering of protons within the simplest real scalar model $\phi^{3}$ (phi-cubed). Although this model has been studied for a very long time, the problem of accounting for the interference contributions for all the possible particle multiplicities observed in experiments is not solved yet. We propose a method which is based on grouping of the interference contributions into sets in such a way that the sum of all interference contributions of each particular set can be calculated with Laplace's method. This approach allowed us to calculate all the interference contributions to the cross sections for multiplicities up to $n \sim 50$ at the energy $\sqrt{s} \sim 50 \mathrm{GeV}$. The obtained models of the energy dependence of the total $p p$ scattering cross section and the inclusive rapidity distribution are in qualitative agreement with the experiment. We also consider the well-known effect of the energy dependence of the shape of inclusive rapidity distribution and propose an explanation for this dependence and consider it exactly as the interference effect.
\end{abstract}

DOI: 10.1103/PhysRevD.101.076021

\section{INTRODUCTION}

When calculating the cross sections of inelastic proton scattering within the multiperipheral model $[1,2]$, it is common to make an assumption that the multi-Regge region [3-7] makes a main contribution to the multidimensional integrals. According to this assumption, the produced particles are strongly ordered in rapidity. The Reggeization approaches [1,2,6,8] use approximations based on this assumption, resulting in the wide variation of the energy-momentum within the integration domain (see the last section in Ref. [9]). At the same time, it is possible to specify, without any assumptions, the integration domain which makes a main contribution to the multidimensional integrals for the scattering cross section. It has been shown (see [9-14]) that the squared modulus of the multiperipheral diagram contribution to the scattering amplitude has quite a distinct conditional maximum given that the energy-momentum law is satisfied. This maximum point may be found either using any numerical method or analytically within a certain approximation [10]. Then one may use Laplace's method [15] to calculate the integrals for the cross sections. The main contribution to the crosssection integral is made by the neighborhood region of the

\section{frumle@ukr.net}

Published by the American Physical Society under the terms of the Creative Commons Attribution 4.0 International license. Further distribution of this work must maintain attribution to the author(s) and the published article's title, journal citation, and DOI. Funded by SCOAP. maximum point; according to Laplace's method, this region is determined by the second derivatives of the logarithm of the integrand at the maximum point. This region is also very different from the multi-Regge one, since it does not force the produced particles to be strongly ordered in rapidity. In particular, when the energy $\sqrt{s}$ is close to the threshold energy for production of the $n$ secondary particles, all the rapidities at the maximum point are approximately equal and close to zero; moreover, the distances between the rapidities at the maximum point increase logarithmically slowly [9] as the energy $\sqrt{s}$ grows and decrease as the $n$ grows. Consequently, the strong ordering of the rapidities for a given multiplicity $n$ may appear only at the energies $\sqrt{s}$ such that the partial cross section of the process with production of the $n$ particles is almost zero.

An absence of the strong ordering of rapidities makes it pointless to consider the multiperipheral diagrams without considering the interference contributions (which represent the various ways of joining of the identical particle lines to the diagram $[9,12])$. Although the values of the interference contributions may be small, their number is too large to be neglected. Moreover, it has been shown (see [12]) that the contribution from the ladder diagram is much smaller compared to the sum of the other contributions. Each interference contribution can be calculated quite easily with Laplace's method [9-14] even for the large number of secondary particles $n$. However, it is problematic to account for the large number of such contributions even for numerical computations. Therefore, the objective of this work is to propose an approximate calculation method which would allow one to calculate the sum of all the 
interference contributions for the processes with a large number of secondary particles $(n \sim 50)$.

The problem of accounting for the interference contributions is considered in Ref. [16] and briefly in Ref. [17]. However, the physical meaning of our approach is significantly different. In Refs. [16,17], the interference contributions have been considered within the approximations which are used in the Amati-Bertocchi-Fubini-StanghelliniTonin (ABFST) model (see [1]), whereas our approach is based on Laplace's method (the difference is analyzed in our previous paper [9]). Note that the assumption about the strong ordering of rapidities is used implicitly in the ABFST model (in order to simplify the integration limits in the integral equation for the $n$-particle contribution to the imaginary part of the scattering amplitude at zero fourmomentum transfer). In addition, in Ref. [16], a calculation of the cross sections is considered only for the cases of the small multiplicities of produced particles, where the simple direct summation of all interference contributions is possible. However, the number of the interference contributions increases rapidly (as $n$ !) as the number of the secondary particles $n$ grows.

It is also necessary to emphasize the difference between our approach and the known models like QGSJET [18] and EPOS [19]. These models are based on the parton-ladder and Pomeron exchange mechanism including AbramovskiiGribov-Kancheli cutting rules (see, e.g., [20,21]). Consequently, they also use the assumption about the dominance of the multi-Regge region. Using such an assumption, one can easily calculate integration over rapidities and obtain the well-known expression for cross section $s^{\alpha(t)}$. The fact that we do not use such an assumption in our approach leads to the necessity of considering the complex rapidity dependence of the scattering amplitude. Whereas in the mentioned models this dependence is not considered, they do account for the interference contributions from the terms with the different positioning of Reggeons relative to the diagram cut. However, they do not consider the structure of the hadronic states within the cut Reggeon. In contrast, in our work, we consider the interference between the final states of the hadrons within the single ladder. Since we do not rely on the mentioned assumption, we do not consider such a ladder, in terms of unitarity condition, as the cut Reggeon.

The Bose-Einstein correlations [22], or the correlations between the four-momenta of the identity particles [23,24], are almost the only interference effect discussed in the literature describing the experiment. This effect is typically considered in the context of the experimental determination of the dimension of the secondary particle radiation region [25-27]. A solution to this problem does not require a detailed description of the dynamics of the inelastic process $[28,29]$. At the same time, it is the dynamics that makes the major difference between our model and the models [30-37] which have been used for the description of the interference effects. Let us discuss this difference.
The main problem when accounting for the interference contributions within the proposed model is that the realvalued integrands of different interference contributions reach their maximum at different points. If all the interference contributions had a common single maximum point, the sum of these interference contributions would also have a single maximum at that point. In this case, one could use Laplace's method to calculate the whole sum of the interference contributions rather than calculating each contribution separately. Moreover, as we show below, in this case the maximum of such a sum would become more pronounced as $n$ grows. As a result, accounting for the interference contributions would not be a problem for an arbitrarily large number of secondary particles. Meanwhile, in our model, the different interference contributions have different maximum points. Consequently, the sum of all interference contributions may have multiple maximum points depending on the energy $\sqrt{s}$ and the number of secondary particles $n$. The number of these maximum points may become so large that the time required for the numerical calculations increases critically. Even when the sum of all interference contributions has only a few maxima, they can be not pronounced enough under certain $\sqrt{s}$ and $n$ to limit to the quadratic terms in Laplace's method. It means that one should take into account the higher-order terms in the Taylor expansion of the integrand logarithm around the maximum point. By contrast, the models considered in Refs. [30-34,38-41] assume that the secondary particles are produced by independent sources, and the Bose-Einstein correlations occur only when the particles propagate from the source to the detector. In this case, different interference contributions are the Fourier transforms of the same function but at different points. As a result, the different interference contributions reach the maximum at the same point, which means that the dynamics in these papers is fundamentally different from the dynamics considered herein. The same is true for the models in Refs. [31,34,35,37,41], in which the problem of the field interacting with the fixed or random (Langevin) source is considered instead of the dynamics of interacting fields. In this case, the secondary particle momentum distribution for an arbitrary number $n$ of the secondary particles is determined by the single function (i.e., by the Fourier transform of the external source); thus, the different interference contributions reach the maximum at the same point. Such models also have an obvious problem associated with the violation of the energy-momentum conservation law caused by space-time translation symmetry breaking. The latter is evidenced by the fact that, according to these models as well as to the models based on the multiRegge kinematics [42], the multiplicity of secondary particles has a Poisson distribution [31]; thus, the particle production processes [43] at different regions of the phase space are independent of each other; consequently, the probability of the production of an arbitrarily large number 
of secondary particles is not zero. In addition, the models with an external source imply source averaging with the corresponding density matrix [33,44-47]. However, the density matrix is actually postulated rather than determined with the time evolution operator obtained within some dynamical model.

From the above, one may conclude that interference effects in the proton-proton scattering must be evident not only in the measurement of the Bose-Einstein correlations, but in any measured quantity. In this paper, we argue that the energy dependence of the shape of inclusive rapidity distribution [48-54] may be considered as an interference effect. The experimental data for the inclusive rapidity distribution are described by the different models [55-64], which, however, do not take into account the interference effects. The manifestation of the interference effects in the inclusive pseudorapidity distribution is considered in Refs. [34,39], though using the above-mentioned approximations in which the different interference contributions reach a maximum value at the same point. By contrast, in this paper, we show that the behavior of the inclusive rapidity (pseudorapidity) distribution shape under the energy $\sqrt{s}$ growth is associated with the change of the distances between the maximum points of the different interference contributions. The mentioned difference between the dynamics considered in this paper and the one considered in Refs. $[34,39]$ becomes apparent from the comparison of the shapes of inclusive rapidity distributions obtained within these models.

In order to focus on the problem of accounting for the interference contributions, let us start with the simplest model $\phi^{3}$. Note, however, that Laplace's method can be applied not only to the $\phi^{3}$ diagrams but also to more complex dynamic models, as shown in Refs. [13,14,65]. Accordingly, the problem of accounting for the interference contributions will also take place within the more realistic models that consider the rapidity dependence of the scattering amplitude.

\section{LAPLACE'S METHOD, $\phi^{3}$ MODEL}

We consider the so-called ladder diagrams for the inelastic $p p$ scattering within the real scalar $\phi^{3}$ model, assuming that the masses of the secondary particles are equal to the pion mass $m_{\pi}$. All the physical quantities used in this work (four-momenta, masses, energies, etc.) are nondimensionalized by the pion mass $m_{\pi}$.

Let $S_{n}$ be the set of all possible permutations of $\mathbb{N}_{n}=\{1,2, \ldots, n\}$. Each diagram of the form in Fig. 1 is characterized by a corresponding permutation $\pi \in S_{n}$. Assuming the one-line notation for the permutations, $\pi(i)$ denotes the index of the secondary particle which is joined to the $i$ th vertex in the corresponding diagram.

Each permutation $\pi \in S_{n}$ specifies the diagram of the form in Fig. 1 and, thus, represents an analytic expressionthe additive contribution to the scattering amplitude

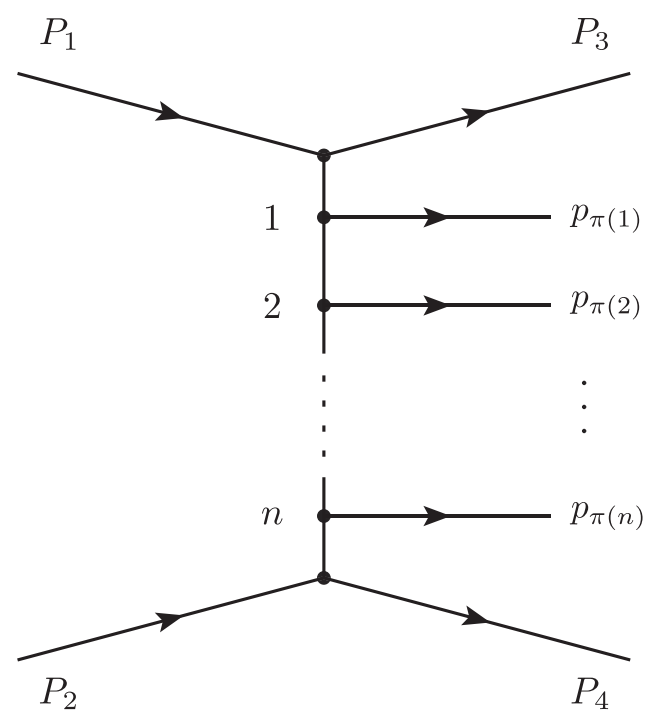

FIG. 1. Feynman ladder diagram within the $\phi^{3}$ model for the inelastic $2 \rightarrow 2+n$ scattering process. The diagram is associated with a permutation $\pi \in S_{n}$.

$$
a_{n}\left(P_{1}, P_{2}, P_{3}, P_{4}, p_{\pi(1)}, \ldots, p_{\pi(n)}\right),
$$

where $P_{1}$ and $P_{2}$ are the four-momenta of the colliding particles in the initial state, $P_{3}$ and $P_{4}$ are the four-momenta of the scattered particles in the final state, and $p_{\pi(1)}, \ldots, p_{\pi(n)}$ are the four-momenta of the secondary particles in the final state. According to the Feynman diagram technique, the scattering amplitude $T_{n}$ of the considered process is the sum of all expressions $a_{n}$ corresponding to the elements of $S_{n}$ :

$$
\begin{aligned}
T_{n} & \left(P_{1}, P_{2}, P_{3}, P_{4}, p_{1}, \ldots, p_{n}\right) \\
& =\sum_{\pi \in S_{n}} a_{n}\left(P_{1}, P_{2}, P_{3}, P_{4}, p_{\pi(1)}, \ldots, p_{\pi(n)}\right) .
\end{aligned}
$$

For convenience, we introduce the following notations. We will consider the scattering process in the center-of-mass reference frame using the right-hand coordinate system in which $z$ axis is codirectional with $\mathbf{P}_{1}$. Here the expressions $a_{n}\left(P_{1}, P_{2}, P_{3}, P_{4}, p_{i_{1}}, \ldots, p_{i_{n}}\right)$ depend on the real particles four-momenta which satisfy the following equations:

$$
\begin{aligned}
& \left(P_{i}\right)_{0}^{2}=M_{i}^{2}+\boldsymbol{P}_{i}^{2}, \\
& \left(p_{i}\right)_{0}^{2}=m^{2}+\boldsymbol{p}_{i}^{2},
\end{aligned}
$$

where $M_{i}$ is the mass of the $i$ th incident particle in the initial state and $m$ is the mass of the secondary particle. The components of $P_{1}$ and $P_{2}$ in the chosen reference frame are $\left(\boldsymbol{P}_{1}\right)_{x}=\left(\boldsymbol{P}_{2}\right)_{x}=0,\left(\boldsymbol{P}_{1}\right)_{y}=\left(\boldsymbol{P}_{2}\right)_{y}=0$, and $\left(\boldsymbol{P}_{1}\right)_{z}=-\left(\boldsymbol{P}_{2}\right)_{z}$. Thus, $P_{1}$ and $P_{2}$ can be uniquely determined by the single quantity $\sqrt{s}=\left(P_{1}\right)_{0}+\left(P_{2}\right)_{0}$ - the total energy of the colliding particles in the center-of-mass reference frame. 
We use the components $\left(\boldsymbol{p}_{i}\right)_{x}$ and $\left(\boldsymbol{p}_{i}\right)_{y}$ of four-momenta and the rapidities $y_{i}$ of the secondary particles as the independent variables which uniquely determine all the four-momenta $p_{i}$. The rapidities $y_{i}$ are determined from the following expression:

$$
\left(\boldsymbol{p}_{i}\right)_{z}=\sqrt{m^{2}+\left(\boldsymbol{p}_{i}\right)_{x}^{2}+\left(\boldsymbol{p}_{i}\right)_{y}^{2}} \sinh \left(y_{i}\right) .
$$

We also introduce another two independent variables $P_{x}^{a}$ and $P_{y}^{a}$ using the following expressions:

$$
\begin{aligned}
& P_{x}^{a}=\left(\boldsymbol{P}_{3}\right)_{x}-\left(\boldsymbol{P}_{4}\right)_{x}, \\
& P_{y}^{a}=\left(\boldsymbol{P}_{3}\right)_{y}-\left(\boldsymbol{P}_{4}\right)_{y} .
\end{aligned}
$$

It follows from Eq. (3) and the energy-momentum conservation law that all the components of $P_{3}$ and $P_{4}$ can be uniquely determined by specifying the $\sqrt{s}, P_{x}^{a}$, and $P_{y}^{a}$ and all the $y_{i},\left(\boldsymbol{p}_{i}\right)_{x}$, and $\left(\boldsymbol{p}_{i}\right)_{y}$.

Finally, instead of $4(n+4)$ variables (the arguments of $a_{n}$ ), we have $3 n+3$ independent variables, so the expression (1) may be rewritten as

$$
\begin{aligned}
& a_{n}\left(\sqrt{s}, y_{1}, \ldots, y_{n},\left(\boldsymbol{p}_{1}\right)_{x}, \ldots,\left(\boldsymbol{p}_{n}\right)_{x},\right. \\
& \left.\left(\boldsymbol{p}_{1}\right)_{y}, \ldots,\left(\boldsymbol{p}_{n}\right)_{y}, P_{x}^{a}, P_{y}^{a}\right) .
\end{aligned}
$$

It is convenient to consider the introduced variables (except $\sqrt{s})$ as the components of some vector $X \in \mathbb{R}^{3 n+2}$ :

$$
\begin{aligned}
& X=\left(y_{1}, \ldots, y_{n},\left(\boldsymbol{p}_{1}\right)_{x}, \ldots,\left(\boldsymbol{p}_{n}\right)_{x},\right. \\
& \left.\left(\boldsymbol{p}_{1}\right)_{y}, \ldots,\left(\boldsymbol{p}_{n}\right)_{y}, P_{x}^{a}, P_{y}^{a}\right) .
\end{aligned}
$$

Finally, it is also natural to introduce the notation for permutations of the components of $X$. To this end, to each permutation $\pi \in S_{n}$ we assign a linear operator $\hat{\pi}: \mathbb{R}^{3 n+2} \rightarrow \mathbb{R}^{3 n+2}$, which can be defined as

$$
\begin{aligned}
\hat{\pi} X=X^{\prime}= & \left(X_{\pi(1)}, \ldots, X_{\pi(n)},\right. \\
& X_{n+\pi(1)}, \ldots, X_{n+\pi(n)}, \\
& X_{2 n+\pi(1)}, \ldots, X_{2 n+\pi(n)}, \\
& \left.X_{3 n+1}, X_{3 n+2}\right),
\end{aligned}
$$

or the same: $(\hat{\pi} X)_{i n+j}=X_{i n+\pi(j)}$ for $i=0 \ldots 2$ and $j=1 \ldots n$, while $(\hat{\pi} X)_{i}=X_{i}$ for $i=3 n+1$ and $3 n+2$. One can see that according to the definition (8) of $\hat{\pi}$, we associate the operator $\hat{\pi}_{2} \hat{\pi}_{1}$ with the composition of permutations $\pi_{1} \circ \pi_{2}$ such that

$$
\begin{aligned}
\left(\hat{\pi}_{2} \hat{\pi}_{1} X\right)_{i n+j} & =\left(\hat{\pi}_{1} X\right)_{i n+\pi_{2}(j)} \\
=(X)_{i n+\pi_{1}\left(\pi_{2}(j)\right)} & =(X)_{i n+\left(\pi_{1} \circ \pi_{2}\right)(j)},
\end{aligned}
$$

where $\hat{\pi}_{i}$ is the operator associated with $\pi_{i}$. Hence, using the introduced notations, the expression (2) can be rewritten in the following way:

$$
T_{n}(\sqrt{s}, X)=\sum_{\pi \in S_{n}} a_{n}(\sqrt{s}, \hat{\pi} X) .
$$

Now let us consider the expression for the partial cross sections $\sigma_{n}$ of inelastic scattering and rewrite this expression using the notations introduced above:

$$
\begin{aligned}
\sigma_{n}= & \frac{(2 \pi)^{4}}{4 n ! I} \int \frac{d \mathbf{P}_{3}}{(2 \pi)^{3} 2\left(P_{3}\right)_{0}} \frac{d \mathbf{P}_{4}}{(2 \pi)^{3} 2\left(P_{4}\right)_{0}} \prod_{i=1}^{n} \frac{d \mathbf{p}_{i}}{(2 \pi)^{3} 2\left(p_{i}\right)_{0}} \\
& \times \delta^{4}\left(P_{1}+P_{2}-P_{3}-P_{4}-\sum_{i=1}^{n} p_{i}\right) \\
& \times\left|T_{n}\left(P_{1}, P_{2}, P_{3}, P_{4}, p_{1}, \ldots, p_{n}\right)\right|^{2}
\end{aligned}
$$

where $I=\sqrt{\left(P_{1} P_{2}\right)^{2}-\left(M_{1}\right)^{2}\left(M_{2}\right)^{2}}$. First, we need to integrate out the energy-momentum-conserving delta function in Eq. (11) and then change the variables of integration. The Jacobian of the transformation and the multipliers appearing as the result of delta function integration can be included in the scattering amplitude $T_{n}$. This procedure is described in detail in Ref. [9]. As a result, the integral (11) takes the following form:

$$
\sigma_{n}(\sqrt{s})=R(n, \sqrt{s}) \int \prod_{i=1}^{3 n+2} d X_{i}\left|T_{n}(\sqrt{s}, X)\right|^{2},
$$

where $R(n, \sqrt{s})=(2 \pi)^{4} / 4 n ! I$.

Now, let us consider the problem of calculations of multidimensional integrals of the form (12).

\section{A. Calculation of the partial cross sections within the $\phi^{3}$ model}

Let us substitute the expression (10) for the scattering amplitude $T_{n}(\sqrt{s}, X)$ in the expression of the partial cross section (12):

$$
\begin{aligned}
\sigma_{n}(\sqrt{s})= & R(n, \sqrt{s}) \\
& \times \int \prod_{i=1}^{3 n+2} d X_{i} \sum_{\pi_{k} \in S_{n}} \sum_{\pi_{l} \in S_{n}}\left[a_{n}^{*}\left(\sqrt{s}, \hat{\pi}_{k} X\right)\right. \\
& \left.\times a_{n}\left(\sqrt{s}, \hat{\pi}_{l} X\right)\right] .
\end{aligned}
$$

Note that one of the two sums over $S_{n}$ in Eq. (13) can be calculated by renaming the variables of integration:

$$
\begin{aligned}
\sigma_{n}(\sqrt{s})= & R(n, \sqrt{s}) n ! \\
& \times \sum_{\pi \in S_{n}} \int \prod_{i=1}^{3 n+2} d X_{i} a_{n}^{*}(\sqrt{s}, X) a_{n}(\sqrt{s}, \hat{\pi} X) .
\end{aligned}
$$

The summands in Eq. (14) are called the interference contributions to the cross section. Each particular permutation $\pi$ specifies some interference contribution which can 


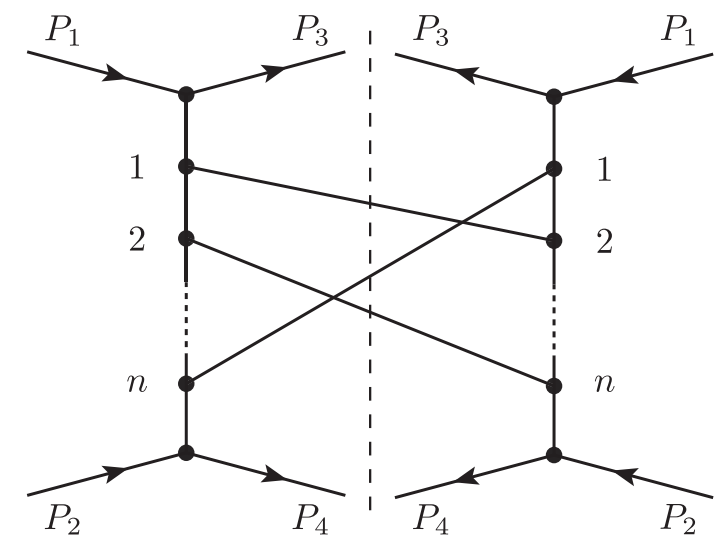

FIG. 2. Cut diagram representing the interference contribution associated with particular permutation $\pi$ such that $\pi(1)=n$, $\pi(2)=1, \ldots, \pi(n)=2$.

be represented by the so-called cut diagram (Fig. 2). The way in which the vertices from both parts of a cut diagram are linked is specified by the permutation $\pi$-according to Eq. (14), the $\pi(i)$ is the index of the vertex in the left part of the diagram which is linked with the $i$ th vertex in the right part of the diagram. Each particular interference contribution can be calculated using Laplace's method (see [9]). This method allows one to easily calculate a multidimensional integral provided its integrand has a single maximum point.

Indeed, it has been shown (see [9]) that the function $a_{n}(\sqrt{s}, X)$ has the single maximum point $X^{(0)}$ at fixed $\sqrt{s}$ :

$$
\begin{aligned}
& X^{(0)}=\left(y_{1}^{(0)}, \ldots, y_{n}^{(0)},\left(\boldsymbol{p}_{1}^{(0)}\right)_{x}, \ldots,\left(\boldsymbol{p}_{n}^{(0)}\right)_{x},\right. \\
& \left.\left(\boldsymbol{p}_{1}^{(0)}\right)_{y}, \ldots,\left(\boldsymbol{p}_{n}^{(0)}\right)_{y}, P_{x}^{a(0)}, P_{y}^{a(0)}\right) .
\end{aligned}
$$

At the same time, the function $a_{n}(\sqrt{s}, \hat{\pi} X)$ has the single maximum point $\hat{\pi}^{-1} X^{(0)}$, where $\hat{\pi}^{-1}$ is the operator (8) associated with permutation $\pi^{-1} \in S_{n}$ inverse of $\pi$; therefore, the product $a_{n}^{*}(\sqrt{s}, X) a_{n}(\sqrt{s}, \hat{\pi} X)$ also has a single maximum point. As a result, Laplace's method can be applied to the calculation of the integrals in expression (14).

The calculation of the total cross sections with Laplace's method even within the simplest $\phi^{3}$ model allows one to obtain the qualitative description of the experimental data (see [9-14]). Moreover, we plan to use Laplace's method to calculate the cross sections within the multiparticle field approach which is based on QCD (see [66,67]).

However, the number of the summands in (14) is $n$ !, which increases dramatically as the energy grows. It becomes impossible to account all of them by calculating each interference contribution separately. So we propose the method which makes it possible to account all the interference contributions for the processes with up to 50 secondary particles.

\section{THE MAIN IDEA OF THE PROPOSED METHOD}

Let us consider a simple example to illustrate the main idea of the proposed method. We say that the maximum points of two functions, each having a single maximum point, are close if the sum of these functions also has a single maximum point. Let $\phi_{a}(x)=\exp \left(-(x-a)^{2}\right)$ be the function parametrized by the parameter $a$ and has single maximum point $x=a$. Now let us consider the sum

$$
g_{a}(x)=\phi_{-a}(x)+\phi_{-a / 2}(x)+\phi_{a / 2}(x)+\phi_{a}(x),
$$

for different values of $a$, and the partial sums $\psi_{-}(x)=$ $\phi_{-a}(x)+\phi_{-a / 2}(x)$ and $\psi_{+}(x)=\phi_{a / 2}(x)+\phi_{a}(x)$. It is easy to see that in the case of $a=0$ the function $g_{0}(x)$ has the single maximum point $x=0$. Then, the distances between the maximum points of summands of $g_{a}(x)$ grow with the parameter $a$. For the values of $a$ in range $[0,1]$, each of the functions $g_{a}(x), \psi_{-}(x)$, and $\psi_{+}(x)$ has a single maximum point, as can be seen in Figs. 3(a) and 3(b). With further growth of $a$, the distances between the maximum points of the summands $\phi$ of $g_{a}(x)$ become

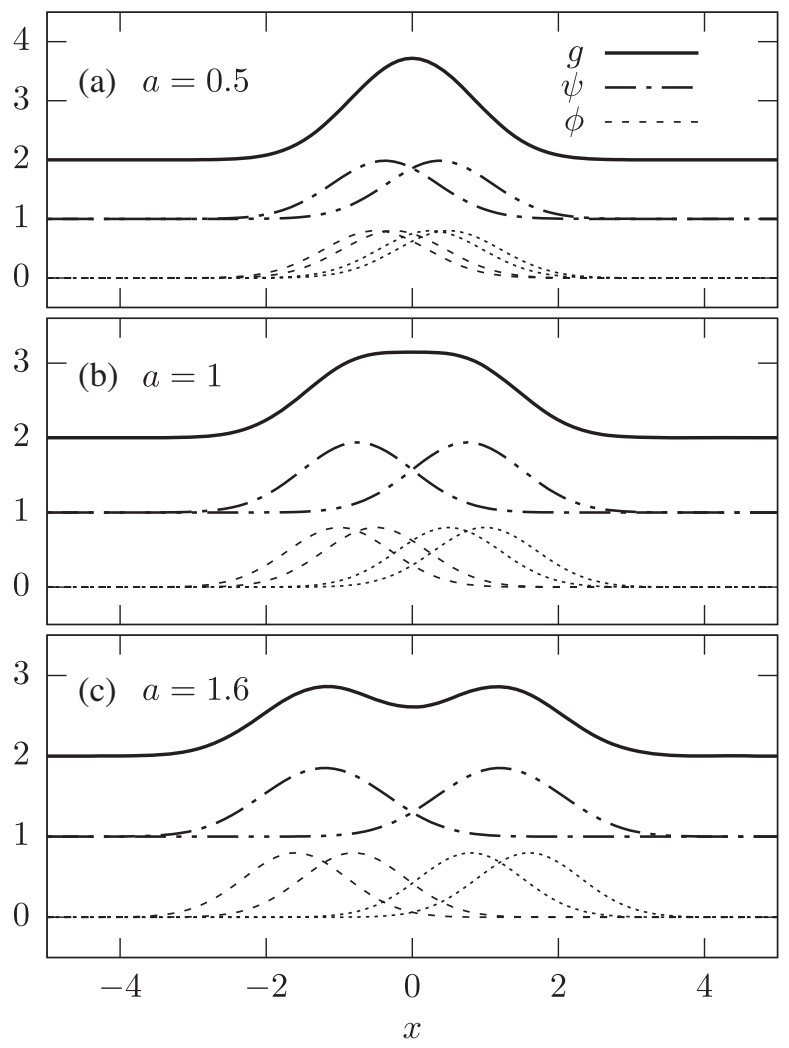

FIG. 3. Plot of the sum $g_{a}(x)$ for the different values of parameter $a$ : (a) $a=0.5$, (b) $a=1.0$, and (c) $a=1.6$. The summands $\phi_{a}(x)$ are represented by the dashed lines, the partial sums $\psi_{-}(x)$ and $\psi_{+}(x)$ by dot-dashed lines, and the total sum $g_{a}(x)$ by the solid line. Note that the functions are normalized and shifted vertically to avoid overlapping. 


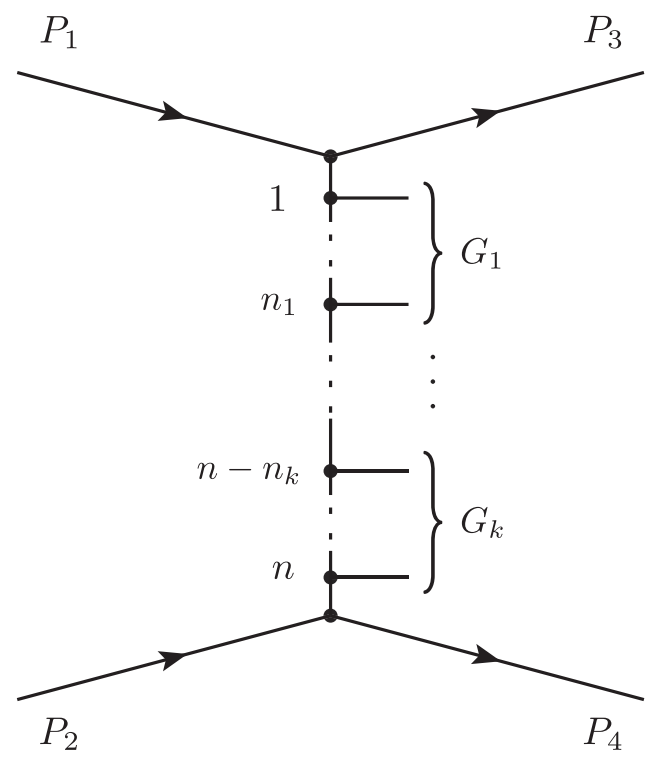

FIG. 4. Grouped vertices.

so large that the single maximum of the function $g_{a}(x)$ splits into two separate maxima [Fig. 3(c)]. At the same time, the partial sums $\psi_{-}(x)$ and $\psi_{+}(x)$ still have the single maximum points.

The similar effect may be observed for the scattering amplitude $T_{n}(\sqrt{s}, X)$ with the energy growth at fixed $n>1$. The function $T_{n}(\sqrt{s}, X)$ has a single maximum at low energies which then splits into a few separate maxima as the energy grows. To show this, let us consider the features of the maximum point $X^{(0)}$ of the function $a_{n}(\sqrt{s}, X)$. At this point, the transverse components of the particles momenta are equal to zero: $\left(\boldsymbol{p}_{i}^{(0)}\right)_{x}=0,\left(\boldsymbol{p}_{i}^{(0)}\right)_{y}=0, P_{x}^{a}=0, P_{y}^{a}=0$, while the rapidities of the secondary particles form the following arithmetic progression (see [9]):

$$
\begin{aligned}
y_{i}^{(0)} & =y_{1}^{(0)}-\Delta_{y}(i-1), \quad \text { where } \\
y_{1}^{(0)} & =\frac{n-1}{2} \Delta_{y}, \\
\Delta_{y} & =\frac{2}{n+1} \operatorname{arcosh}\left(\frac{\sqrt{s}-n}{2 M}\right) .
\end{aligned}
$$

As seen from Eq. (17), $y_{i}=-y_{n-i}$. Now consider the expression for the scattering amplitude $T_{n}(\sqrt{s}, X)$, i.e., the sum (10), taking into account the mentioned features of $X^{(0)}$. As we have already shown in the previous section, each summand of this sum [i.e., the function $a_{n}(\sqrt{s}, \hat{\pi} X)$ ] is associated with some permutation $\pi$ and reaches its maximum at the single point $\hat{\pi}^{-1} X^{(0)}$. The distance $\rho$ between the maximum points of two such summands (associated with $\pi_{1}$ and $\pi_{2}$ ) is defined by the following expression:

$$
\rho\left(\hat{\pi}_{1}^{-1} X^{(0)}, \hat{\pi}_{2}^{-1} X^{(0)}\right)=\left|\Delta_{y}\right| \sqrt{\sum_{i=1}^{n}\left[\pi_{1}^{-1}(i)-\pi_{2}^{-1}(i)\right]^{2}} .
$$

Here we have taken into account that $X_{i}=0$ for $n<i<3 n+2$. Given the fixed number of secondary particles $n$, one can see from Eq. (18) that the common difference $\left|\Delta_{y}\right|$ of the rapidity arithmetic progression tends to zero as the energy $\sqrt{s}$ goes to the threshold value $2 M+n$. In this case, all the summands are close, so the whole sum (10) has a single maximum point; it means that one can apply Laplace's method to calculate the integral (12), thus taking into account all the interference contributions in the simple way.

However, the $\left|\Delta_{y}\right|$ slowly increases as the energy grows, and at some point the distances between the summands in Eq. (10) become so large that the whole sum acquires several maxima. In other words, the single maximum of the scattering amplitude $T_{n}(\sqrt{s}, X)$ splits into a few separate maxima as the energy grows. We have already faced the similar effect for the one-dimensional function in the beginning of this section. In this case, Laplace's method cannot be applied to the calculation of the integral (12), since its integrand has several maxima. One could still consider the sum (14) and calculate each interference contribution with Laplace's method, but this way it is impossible to calculate all the interference contributions for the processes with a large number of secondary particles.

The main idea of the proposed method of accounting for the interference contributions is to apply Laplace's method not to each interference contribution separately, but to the sums of the interference contributions whose integrands have close maximum points. The integrand of such a sum has a single maximum point, so the whole sum can be calculated with Laplace's method. Although the scattering amplitude $T_{n}(\sqrt{s}, X)$ acquires several maxima as the energy grows, we can split the sum (10) into subsums, each having a single maximum point. To this end, we group the neighboring vertices of the diagram into $k$ groups. Then we group all the permutations $\pi \in S_{n}$ into subsets $I_{1}, I_{2}, \ldots$ in such a way that any two permutations belong to the same subset if and only if they specify the diagrams in which the lines of the secondary particles are joined to the same groups of the vertices regardless of the order within the groups. So the diagrams specified by the permutations belonging to the same subset $I_{i}$ differ only in the order of joining inside the groups of vertices. Therefore, according to Eqs. (17) and (18), we expect the functions associated with such diagrams to have close maximum points. Thus, for each subset $I_{i}$, we consider the sum $b_{i}(\sqrt{s}, X)=$ $\sum_{\pi \in I_{i}} a_{n}(\sqrt{s}, \hat{\pi} X)$. For any given values of $n$ and $\sqrt{s}$, we select such a number, and the sizes of the groups that make each of the functions $b_{1}, b_{2}, \ldots$ have a single maximum point. As a result, one can use Laplace's method to calculate the sum of the interference contributions associated with each subset $I_{i} \subset S_{n}$ rather than calculating the interference contribution associated with each permutation $\pi \in S_{n}$. 
Such an approach reduces greatly the number of calculations and allows one to account for all the interference contributions for the processes with high production of the secondary particles (up to 50 secondary particles at $72 \mathrm{GeV}$ at present). The specific implementation of the proposed idea will be described in the next section in detail.

\section{A. Grouping the vertices of the diagram}

Let us group the neighboring vertices of the diagram in Fig. 1 into the $k$ groups (nonempty sets), each containing $n_{i}$ vertices (Fig. 4). For this purpose, we consider the sequence of sets $G_{1}, G_{2}, \ldots, G_{k}$, each specifying a corresponding group. The set $G_{i}$ contains the indices of vertices that are grouped into the $i$ th group:

$$
G_{i}=\left\{\nu \in \mathbb{N}_{n} \mid \sum_{k=1}^{i-1} n_{k}<\nu \leq \sum_{k=1}^{i} n_{k}\right\}
$$

for $1 \leq i \leq k$, where $n_{i}$ is the number of vertices in the $i$ th group.

The lines of the secondary particles in the connected diagram specified by a permutation $\pi$ are distributed among the groups of the vertices in some way depending on the permutation. Thus, for any permutation $\pi \in S_{n}$ we can also consider the sets $\pi\left(G_{1}\right), \pi\left(G_{2}\right), \ldots, \pi\left(G_{k}\right)$, where $\pi\left(G_{i}\right)=$ $\left\{\pi(\nu) \mid \nu \in G_{i}\right\}$ is the set containing the indices of the secondary particles which are joined to the vertices of the $i$ th group in the diagram specified by the permutation $\pi$. It allows us to introduce the equivalence relation $\sim$ on the permutations set $S_{n}$ in the following way:

$\pi_{i} \sim \pi_{j} \Leftrightarrow \quad \forall l \in[1 \ldots k], \quad \pi_{i}\left(G_{l}\right)=\pi_{j}\left(G_{l}\right)$,

which means that any two permutations $\pi_{i}$ and $\pi_{j}$ are equivalent if they specify the diagrams in which the external lines of the secondary particles are joined to the same groups of the vertices regardless of the joining order inside the groups (Fig. 5). As a result, the permutations set $S_{n}$ may be split into the equivalence classes $[\pi]=$ $\left\{\pi_{i} \in S_{n} \mid \pi_{i} \sim \pi\right\}$. In other words, the introduced equivalence relation $\sim$ provides the partition $S_{n} / \sim$ of the underlying set $S_{n}$, where $S_{n} / \sim$ is the quotient set of the permutations set $S_{n}$ by $\sim$; i.e., the set contains all the equivalence classes $[\pi]$ :

$$
S_{n}=\bigcup_{[\pi] \in S_{n} / \sim}[\pi] .
$$

Taking into account (21), the expression (10) may be rewritten as

$$
T_{n}(\sqrt{s}, X)=\sum_{[\pi] \in S_{n} / \sim}\left[\sum_{\pi_{i} \in[\pi]} a_{n}\left(\sqrt{s}, \hat{\pi}_{i} X\right)\right] .
$$

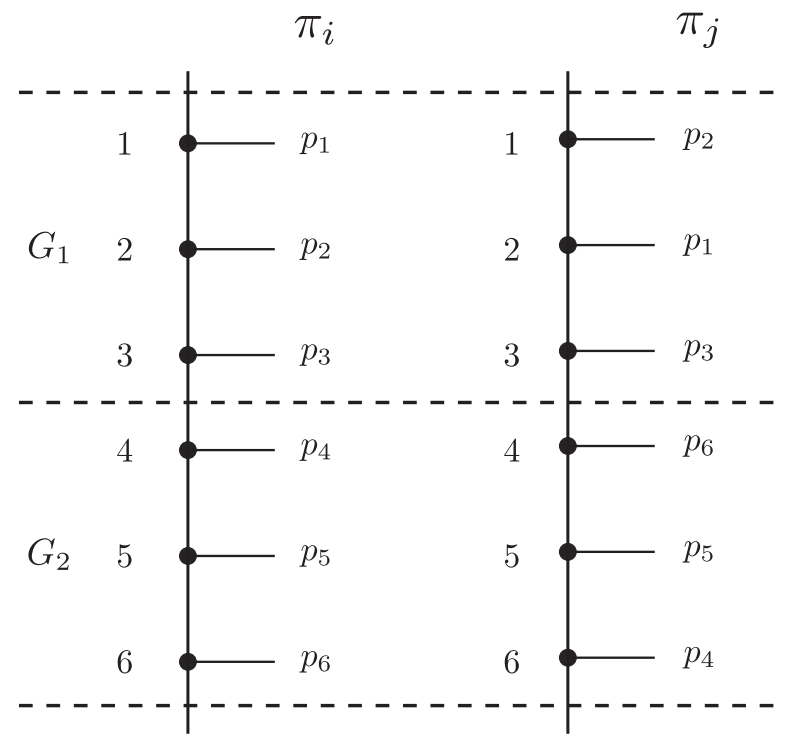

FIG. 5. Example of equivalent permutations $\pi_{i}$ and $\pi_{j}$ given $n=6, G_{1}=\{1,2,3\}$, and $G_{2}=\{4,5,6\}$. Here $\pi_{i}\left(G_{1}\right)=$ $\pi_{j}\left(G_{1}\right)=\{1,3,5\} \quad$ and $\pi_{i}\left(G_{2}\right)=\pi_{j}\left(G_{2}\right)=\{2,4,6\}$, which imply that $\pi_{i} \sim \pi_{j}$.

Now let us consider the auxiliary function $A_{n}(\sqrt{s}, X)$ :

$$
A_{n}(\sqrt{s}, X)=\sum_{e \in[\epsilon]} a_{n}(\sqrt{s}, \hat{e} X),
$$

where $[\epsilon]$ is the equivalence class containing the permutations equivalent to the identity permutation $\epsilon$ such that $\epsilon(i)=i$ for all $i \in[1 \ldots n]$. The maximum distance between the maximum points of summands in Eq. (23) may be significantly reduced compared to the summands of Eq. (10) by increasing the number of groups $k$ and decreasing their sizes $n_{1}, n_{2}, \ldots n_{k}$. Therefore, for any given values of $n$ and $\sqrt{s}$, one may select such a number of the groups $k$ and sizes of the groups $n_{1}, n_{2}, \ldots n_{k}$ that make function $A_{n}(\sqrt{s}, X)$ have a single maximum point. It may be shown that for any permutation $\pi_{i} \in[\pi]$ there exists a permutation $e \in[\epsilon]$ such that $\pi_{i}=\pi \circ e$, where $\pi$ is the representative (any member) of the class $[\pi]$; taking this into account and also (9), let us represent the scattering amplitude (22) in terms of $A_{n}(\sqrt{s}, X)$ :

$$
\begin{aligned}
T_{n}(\sqrt{s}, X) & =\sum_{[\pi] \in S_{n} / \sim}\left[\sum_{e \in[\epsilon]} a_{n}(\sqrt{s}, \hat{e}(\hat{\pi} X))\right] \\
& =\sum_{[\pi] \in S_{n} / \sim} A_{n}(\sqrt{s}, \hat{\pi} X),
\end{aligned}
$$

where $\hat{\pi}$ is the permutation operator (8) associated with the representative of $[\pi]$. As a result, the integral (12) may also be written in terms of $A_{n}(\sqrt{s}, X)$ : 


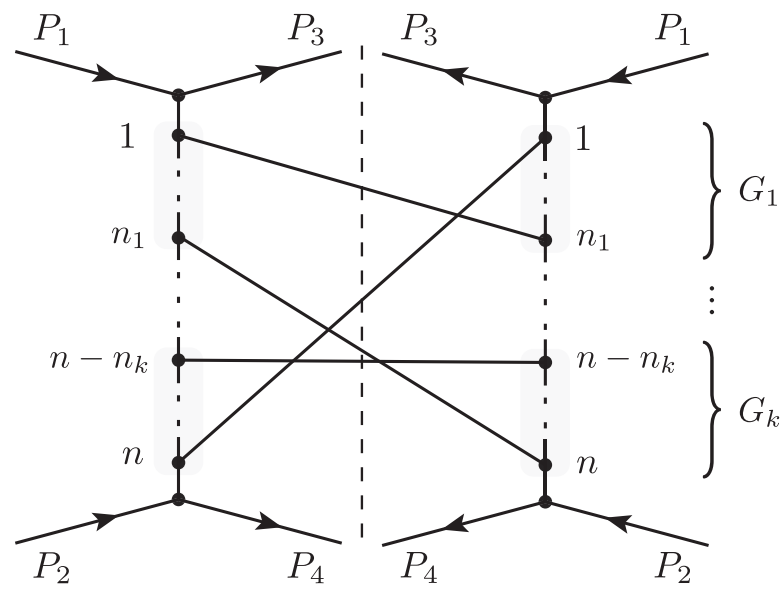

FIG. 6. Cut diagram in which the vertices of both parts are grouped into $k$ groups.

$$
\begin{aligned}
\sigma_{n}(\sqrt{s})= & R(n, \sqrt{s}) \\
& \times \int \prod_{i=1}^{3 n+2} d X_{i}\left[\sum_{\left[\pi_{i}\right] \in S_{n} / \sim} A_{n}^{*}\left(\sqrt{s}, \hat{\pi}_{i} X\right)\right. \\
& \left.\times \sum_{\left[\pi_{k}\right] \in S_{n} / \sim} A_{n}\left(\sqrt{s}, \hat{\pi}_{k} X\right)\right] .
\end{aligned}
$$

One of the two summations in the integral (25) may be calculated by renaming the variables of integration as was done between Eqs. (13) and (14). Note that in this case the number of the elements in the set of summation $S_{n} / \sim$ is $n ! / n_{1} ! n_{2} ! \ldots n_{k} !$

$$
\begin{aligned}
\sigma_{n}(\sqrt{s})= & R(n, \sqrt{s}) \frac{n !}{n_{1} ! n_{2} ! \ldots n_{k} !} \\
& \times \sum_{[\pi] \in S_{n} / \sim} \int \prod_{i=1}^{3 n+2} d X_{i} A_{n}^{*}(\sqrt{s}, X) A_{n}(\sqrt{s}, \hat{\pi} X) .
\end{aligned}
$$

Taking into account that the function $A_{n}(\sqrt{s}, X)$ has a single maximum point, Laplace's method may be used to calculate the summands of Eq. (26). The number of the summands in Eq. (26), which is equal to $\left|S_{n} / \sim\right|=$ $n ! / n_{1} ! n_{2} ! \ldots n_{k} !$, may be significantly reduced by selecting the number of groups $k$ such that $k \ll n$. Each summand of Eq. (26) can also be represented by the corresponding cut diagram in Fig. 6. Hence, according to Eq. (26), it is sufficient to consider only a single permutation from each equivalence class $[\pi]$ rather then all permutations of $S_{n}$ in order to calculate all the interference contributions to the cross section $\sigma_{n}$. Moreover, we will show that there are many similar summands in Eq. (26) which may be easily calculated by the corresponding weight factors.

Let us consider a summand of Eq. (26) associated with a class $\left[\pi_{i}\right]$. Then change the integration variables: $X \rightarrow \hat{e} X$, where $e$ is a permutation belonging to class $[\epsilon]$. Note that the function $A_{n}$ is symmetric with respect to such a transition: $A_{n}(\sqrt{s}, \hat{e} X)=A_{n}(\sqrt{s}, X)$ :

$$
\begin{aligned}
\int & d X A_{n}^{*}(\sqrt{s}, X) A_{n}\left(\sqrt{s}, \hat{\pi}_{i} X\right) \\
= & \int d(\hat{e} X) A_{n}^{*}(\sqrt{s}, \hat{e} X) A_{n}\left(\sqrt{s}, \hat{\pi}_{i} \hat{e} X\right) \\
= & \int d X A_{n}^{*}(\sqrt{s}, X) A_{n}\left(\sqrt{s}, \hat{\pi}_{l} X\right) .
\end{aligned}
$$

According to the definition of permutation operator (8), the operator $\hat{\pi}_{l}=\hat{\pi}_{i} \hat{e}$ is assigned to the permutation $\pi_{l}=e \circ \pi_{i}$. It may be shown that the permutations $\pi_{i}$ and $e$ may be selected such that $\pi_{i}$ and $\pi_{l}=e \circ \pi_{i}$ belong to the different equivalence classes $\left[\pi_{i}\right]$ and $\left[\pi_{l}\right]$. As a result, one can see from Eq. (27) that there are two similar contributions to the sum (26) associated with the different equivalence classes.

In other words, the definition of the function $A_{n}$ implies that both parts of the cut diagram in Fig. 6 are symmetric with respect to the order of joining inside the groups of vertices. Consequently, the permutations which establish the same number of connections between the groups of vertices differ only in the order of linking inside the groups and, therefore, represent similar contributions to the sum (26). To clarify this statement, let us consider the number of connections $m_{i j}$ between the $i$ th group in the left part and the $j$ th group in the right part of the cut diagram (6) specified by a permutation $\pi$ :

$$
m_{i j}=\left|\pi\left(G_{i}\right) \cap G_{j}\right|,
$$

where $|\cdot|$ denotes the number of elements in the set (i.e., the power of the set). In this way, each equivalence class $[\pi]$ may be characterized by a matrix $m$ of size $k \times k$ whose elements are defined by Eq. (28). If any two classes $\left[\pi_{i}\right]$ and $\left[\pi_{j}\right]$ are characterized by the same matrix $m$, then they specify the equivalent cut diagrams, which means that they correspond to similar contributions to the sum (26), because one of these diagrams can be obtained from another by rearranging the vertices inside the groups.

In order to consider all the unique summands of Eq. (26), we consider the set $M$ of all possible matrices $m$ of size $k \times k$ such that the sum of the elements of the $i$ th row (column) is equal to the number of vertices in the $i$ th group:

$$
M=\left\{m \in M_{k, k}(\mathbb{N}): \sum_{j=1}^{k} m_{i j}=\sum_{j=1}^{k} m_{j i}=n_{i}\right\}
$$

Each matrix $m$ represents a summand in Eq. (26). All the summands similar to this one can be accounted by weight factor $W_{m}$ uniquely determined by the elements of the matrix $m$. To calculate the summand specified by the matrix $m$, one needs to link the vertices of the cut diagram in a way specified by the matrix (Fig. 7). As a result, one obtains the 


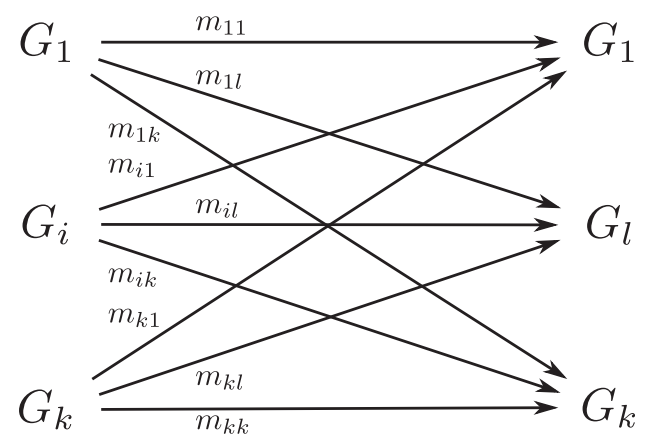

FIG. 7. The number of the connections $m_{i j}$ between the groups in the cut diagram specified by some permutation $\pi$.

permutation $\pi_{m}$ such that $\left|\pi_{m}\left(G_{i}\right) \cap G_{j}\right|=m_{i j}$, which makes it possible to calculate the summand associated with the class $\left[\pi_{m}\right]$. The weight factor $W_{m}$ may be calculated by counting the number of different equivalence classes $[\pi]$ characterized by the same matrix $m$. The number of such classes is equal to the number of all possible ways in which one can group the elements of the sets $G_{1}, G_{2}, \ldots, G_{n}$ into $k$ sets $G_{1}^{\prime}, G_{2}^{\prime}, \ldots, G_{k}^{\prime}$ such that $\left|G_{i} \cap G_{j}^{\prime}\right|=m_{i j}$, where $i, j \in[1 \ldots k]$, so that each set $G_{j}^{\prime}$ contains exactly $m_{i j}$ elements from the set $G_{i}$ :

$$
W_{m}=\prod_{i=1}^{k} \frac{n_{i} !}{\prod_{j=1}^{k} m_{i j} !}
$$

Finally, the expression for the partial cross section may be rewritten as

$$
\begin{aligned}
\sigma_{n}(\sqrt{s})= & R(n, \sqrt{s}) \frac{n !}{n_{1} ! n_{2} ! \ldots n_{k} !} \\
& \times \sum_{m \in M} W_{m} \int \prod_{i=1}^{3 n+2} d X_{i} A_{n}^{*}(\sqrt{s}, X) A_{n}\left(\sqrt{s}, \hat{\pi}_{m} X\right) .
\end{aligned}
$$

Note that the number of summands in Eq. (31) may be much smaller compared to the sum (11). At the same time, Laplace's method may be used to calculate each particular summand of the sum (31).

It remains now to consider in detail all the components necessary to apply Laplace's method within the proposed approach for the calculation of interference contributions.

First, we consider the feature of the maximum point of the function $A_{n}(\sqrt{s}, X)$. We have already mentioned the features of the maximum point of $a_{n}(\sqrt{s}, X)$ in Sec. III. Since the function $a_{n}(\sqrt{s}, X)$ grows as $\left(\mathbf{p}_{\perp}\right)_{i} \rightarrow 0$ and $\mathbf{P}_{\perp}^{a} \rightarrow 0$, the function (23) reaches the maximum value at a point $\chi^{(0)}$ where $\left(\mathbf{p}_{\perp}\right)_{i}=0, i=1 \ldots n$ and $\mathbf{P}_{\perp}^{a}=0$; so $\chi_{i}^{(0)}=0$ for $n<i \leq 3 n+2$.
As we mentioned above, we group the vertices of the diagram in a way for the function $A_{n}(\sqrt{s}, X)$ to have a single maximum point. Taking also into account that $A_{n}(\sqrt{s}, \hat{e} X)=A_{n}(\sqrt{s}, X)$ for any $e \in[\epsilon]$, one can conclude that

$$
\hat{e} \chi^{(0)}=\chi^{(0)}
$$

for any $e \in[\epsilon]$. This important feature of $\chi^{(0)}$ allows one to calculate the value of function $A_{n}$ together with its second derivatives at the maximum point in a simple way:

$$
\begin{aligned}
A_{n}^{0} & =A_{n}\left(\sqrt{s}, \chi^{(0)}\right)=\sum_{e \in[\epsilon]} a_{n}\left(\sqrt{s}, \hat{e} \chi^{(0)}\right) \\
& =\left(\prod_{i=1}^{k} n_{i} !\right) a_{n}\left(\sqrt{s}, \chi^{(0)}\right) .
\end{aligned}
$$

Let us consider the first $n$ components of the vector of derivatives $\partial A_{n} / \partial X_{i}$ of $A_{n}$ at the maximum point $\chi^{(0)}$ assuming that $\chi^{(0)}$ satisfies Eq. (32). We denote these components by $\partial A_{n} / \partial y_{i}$ :

$$
\begin{aligned}
\frac{\partial A_{n}}{\partial y_{i}}\left(\sqrt{s}, \chi^{(0)}\right) & =\sum_{e \in[\epsilon]} \frac{\partial a_{n}}{\partial y_{e^{-1}(i)}}\left(\sqrt{s}, \hat{e} \chi^{(0)}\right) \\
& =\frac{\prod_{j=1}^{k} n_{j} !}{n_{g(i)}} \sum_{j \in G_{g(i)}} \frac{\partial a_{n}}{\partial y_{j}}\left(\sqrt{s}, \chi^{(0)}\right),
\end{aligned}
$$

where $g(i)$ is the index of the group containing the index $i$ (so that $\left.i \in G_{g(i)}\right)$ and $e^{-1}$ is the inverse permutation of $e$, i.e., such that $e^{-1} \circ e=\epsilon$. The derivatives $\partial A_{n} / \partial\left(p_{x}\right)_{i}$ and $\partial A_{n} / \partial\left(p_{y}\right)_{i}$ are calculated in the same way as $\partial A_{n} / \partial y_{i}$. For the last two components of $\partial A_{n} / \partial X_{i}$, we have

$$
\frac{\partial A_{n}}{\partial X_{i}}(\sqrt{s}, X)=\prod_{j=1}^{k} n_{j} ! \frac{\partial a_{n}}{\partial X_{i}}(\sqrt{s}, X),
$$

where $i=3 n+1,3 n+2$.

The matrix of the second derivatives $\partial^{2} A_{n} /$ $\partial X_{i} \partial X_{j}\left(\sqrt{s}, \chi^{(0)}\right)$ may be split into blocks with respect to the variables: $y_{i}$ and $\left(\mathbf{p}_{x}\right)_{x},\left(\mathbf{p}_{x}\right)_{y}$. Then each block may be considered separately. Let us start with the block containing the derivatives $\partial^{2} A_{n} / \partial y_{i} \partial y_{j}\left(\sqrt{s}, \chi^{(0)}\right)$ which we denote briefly by $D_{i j}^{(0)}$ :

$$
D_{i j}^{(0)}=\sum_{e \in[\epsilon]} \frac{\partial^{2} a_{n}}{\partial y_{e^{-1}(i)} \partial y_{e^{-1}(j)}}\left(\sqrt{s}, \hat{e} \chi^{(0)}\right) .
$$

In order to calculate the sum (36), we denote the derivatives $\partial^{2} a_{n} / \partial y_{i} \partial y_{j}\left(\sqrt{s}, \chi^{(0)}\right)$ by $d_{i j}^{(0)}$ and consider the following cases keeping in mind that $\chi^{(0)}$ satisfies Eq. (32). 
(1) $g(i)=g(j)$ and $i=j$ :

$$
D_{i j}^{(0)}=\frac{\prod_{l=1}^{k} n_{l} !}{n_{g(i)}} \sum_{i_{1} \in G_{g(i)}} d_{i_{1} i_{1}}^{(0)}
$$

(2) $g(i)=g(j)$ and $i \neq j$ :

$$
D_{i j}^{(0)}=\frac{\prod_{l=1}^{k} n_{l} !}{\left(n_{g(i)}-1\right) n_{g(i)}} \sum_{\substack{i_{1} i_{i} \in G_{g(i)} \\ i_{1} \neq i_{2}}} d_{i_{1} i_{2}}^{(0)}
$$

(3) $g(i) \neq g(j)$ :

$$
D_{i j}^{(0)}=\frac{\prod_{l=1}^{k} n_{l} !}{n_{g(i)} n_{g(j)}} \sum_{\substack{i_{1} \in G_{g(i)} \\ i_{2} \in G_{g(j)}}} d_{i_{1} i_{2}}^{(0)} .
$$

The rest of the blocks containing the derivatives $\partial^{2} A_{n} /$ $\partial\left(\mathbf{p}_{x}\right)_{i} \partial\left(\mathbf{p}_{x}\right)_{j}\left(\sqrt{s}, \chi^{(0)}\right)$ and $\partial^{2} A_{n} / \partial\left(\mathbf{p}_{y}\right)_{i} \partial\left(\mathbf{p}_{y}\right)_{j}\left(\sqrt{s}, \chi^{(0)}\right)$ may be calculated in the same way as Eqs. (37)-(39). Note that, according to Eqs. (37)-(39), all the mixed derivatives $\partial^{2} A_{n} / \partial y_{i} \partial\left(\mathbf{p}_{x}\right)_{j}\left(\sqrt{s}, \chi^{(0)}\right)$ and $\partial^{2} A_{n} / \partial y_{i} \partial\left(\mathbf{p}_{y}\right)_{j} \times$ $\left(\sqrt{s}, \chi^{(0)}\right)$ are equal to zero, since the derivatives $\partial^{2} a_{n} /$ $\partial y_{i} \partial\left(\mathbf{p}_{x}\right)_{j}\left(\sqrt{s}, X^{(0)}\right)$ and $\partial^{2} a_{n} / \partial y_{i} \partial\left(\mathbf{p}_{y}\right)_{j}\left(\sqrt{s}, X^{(0)}\right)$ are equal to zero (see [9]).

Thus, we obtain all the components necessary to calculate the sum (31) with Laplace's method.

\section{RESULTS}

We applied the proposed method within the $\phi^{3}$ model to calculate the energy dependence of the $p p$ scattering total cross section and the inclusive rapidity distribution for inelastic $p p$ scattering. The models presented in Figs. 8 and 9 are obtained at the value of effective coupling constant $L=29$ (the expression for $L$ is the same as in Ref. [9], p. 876). As we can see from Figs. 8 and 9, a comparison with the experimental data shows that the proposed approach allows us to obtain the theoretical predictions that are in qualitative agreement with the experimental data even within the simplest model. The following expression has been used for the calculations of inclusive rapidity distribution:

$\left.\frac{\partial \sigma_{i n c l}}{\partial y}(\sqrt{s}, y) \propto \sum_{n=1}^{N(\sqrt{s})} \sum_{i=1}^{n} \int \prod_{\substack{j=1 \\ j \neq i}}^{3 n+2} d X_{j}\left|T_{n}(\sqrt{s}, X)\right|^{2}\right|_{X_{i}=y}$,

where $N(\sqrt{s})$ is for the maximum available number of the secondary particles (each having mass $m$ ) which may be produced as the result of inelastic scattering at a fixed energy value $\sqrt{s}$.

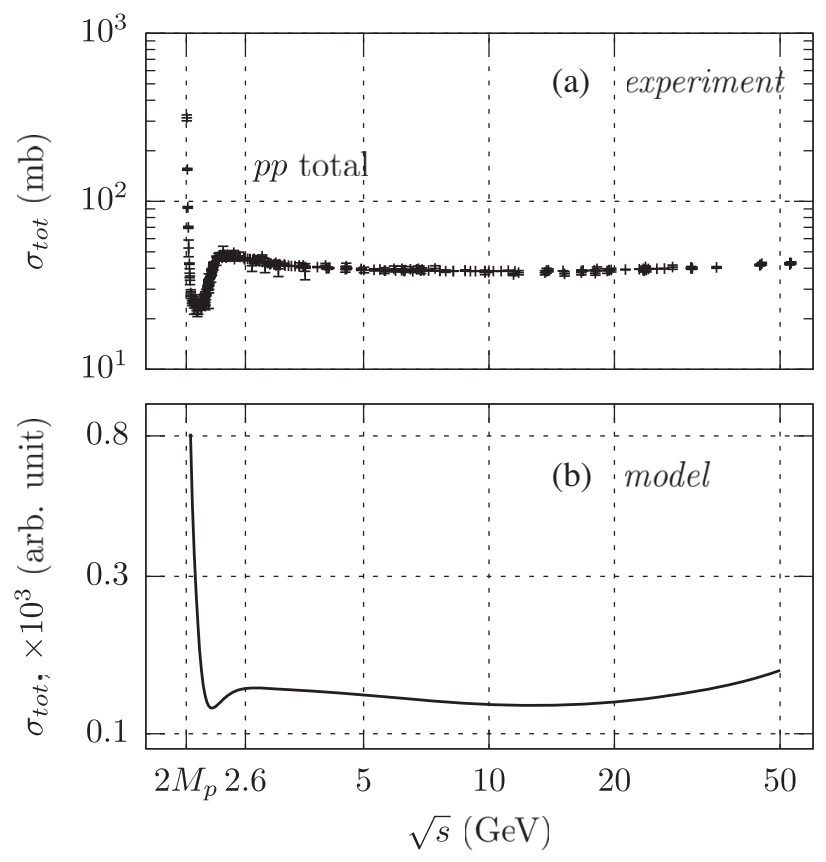

FIG. 8. The energy dependence of the total cross section of $p p$ scattering. Experimental data [68] (a) and the dependency calculated with the proposed method taking into account all the interference contributions for the diagrams with up to $n=50$ secondary particles (b). $M_{p}$ is the proton mass.

It is of interest to consider the peak behavior in Fig. 9. At low energies, one can observe the single peak at rapidities (pseudorapidities) close to zero. However, this peak becomes less pronounced and transforms into the so-called rapidity plateau as the energy $\sqrt{s}$ grows. With further energy growth, the plateau splits into the two separate peaks at nonzero points located symmetrically about zero.

The effect described above may be explained by analyzing the behavior of the maximum points of the scattering amplitude absolute value $\left|T_{n}(\sqrt{s}, X)\right|^{2}$ with the energy growth. Note that the function $\left|T_{n}(\sqrt{s}, X)\right|$ has the same maximum points as $T_{n}(\sqrt{s}, X)$, since the scattering amplitude (10) within the $\phi^{3}$ model is the real-valued function, except for the constant complex factor which may be neglected:

$$
T_{n}(\sqrt{s}, X)=\sum_{\pi \in S_{n}} a_{n}(\sqrt{s}, \hat{\pi} X)
$$

Let us consider Eq. (41) at the values of energy close to the inelastic threshold energy $\sqrt{s} \gtrsim 2 M+m$. In this case, according to Eq. (17), for any $n=1, \ldots, N(\sqrt{s})$, the rapidities of the secondary particles $y_{1}^{(0)}, y_{2}^{(0)}, \ldots, y_{n}^{(0)}$ at the maximum point of the function $a_{n}(\sqrt{s}, X)$ are close to zero. Thus, the summands in Eq. (41) have close maximum points, so the function $T_{n}(\sqrt{s}, X)$ has the single maximum point $\zeta^{(0)}$. It means also that the single group $(k=1)$ is enough to make Eq. (23) the function $A_{n}(\sqrt{s}, X)$ [which in 

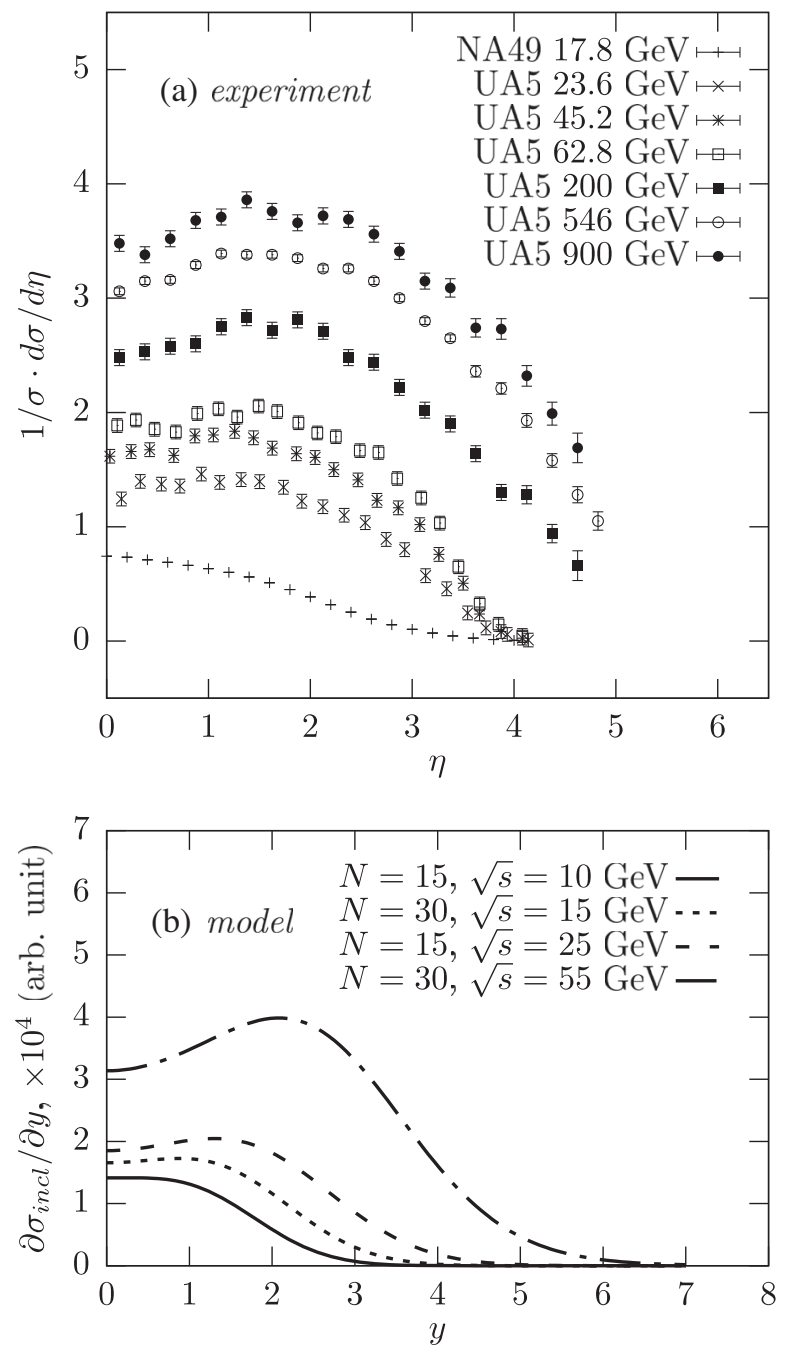

FIG. 9. Rapidity (pseudorapidity) distributions in $p p$ and $p \bar{p}$ collisions at various center of mass energies. Experimental data [50,69] (a). Rapidity distribution calculated with the proposed method within the $\phi^{3}$ model (b). Here $N$ denotes the maximum number of the secondary particles for which all the interference contributions are calculated.

this case is identically equal to $\left.T_{n}(\sqrt{s}, X)\right]$ have a single maximum point. Taking this into account and also Eqs. (17) and (32), one may conclude that $\zeta^{(0)}=(0, \ldots, 0)$. Hence, the most probable inelastic processes at low energies are the processes in which all the secondary particles have nearzero rapidities; i.e., the mean value of the vector of secondary particle rapidities at low energies is equal to zero. As a result, the inclusive rapidity distribution has the single pronounced maximum at zero.

The continuous growth of the energy $\sqrt{s}$ increases the distances between the maximum points of the summands in Eq. (41). As a result, the single maximum of $T_{n}(\sqrt{s}, X)$ becomes less pronounced; i.e., the absolute values of eigenvalues $\left|\lambda_{i}\right|$ of the Hessian matrix (with respect to $y$ ) of the function $T_{n}$ at the maximum point $\zeta^{(0)}$ decrease and tend to zero. It means that the variances of the rapidities of the secondary particles are increasing with energy growth while the mean values remain zero. Accordingly, the single maximum of the inclusive rapidity distribution transforms smoothly into the so-called rapidity plateau.

The further energy growth makes the maximum points of the summands in Eq. (41) move away from each other so far that the single maximum of the $T_{n}(\sqrt{s}, X)$ observed at lower energies splits into a few separate maxima. As a result, the eigenvalues $\lambda_{i}$ become positive, while the first derivatives are still equal to zero, which indicates that there is a local minimum of the function $T_{n}(\sqrt{s}, X)$ at the point $\zeta^{(0)}$. It means that, as the energy grows, the processes in which the secondary particles have nonzero rapidities become more probable than the processes in which all the secondary particles have zero rapidities. Hence, the maxima of the inclusive rapidity distribution move away from zero. Moreover, the maximum points of the function $T_{n}$ are symmetrically distributed about zero due to the symmetry of the considered physical system with respect to the inversion of the collision axis. As a result, the maxima of the inclusive rapidity distribution at high energies are also symmetrically distributed about zero, which is in agreement with the experiment.

\section{CONCLUSIONS}

We developed the method of accounting for the interference contributions to inelastic scattering cross sections. Using this method, we obtained the models of energy dependence of the total cross section and rapidity distribution for the proton-proton scattering at energies up to $72 \mathrm{GeV}$ and multiplicities up to 50. This model reproduces the experimental data only qualitatively, which can be explained by the fact that the modeling has been performed within the $\phi^{3}$ (phi-cubed) model-the simplest dynamical model with the scalar field.

The obtained results indicate that it would be appropriate to use the proposed method with a more complex model, for instance, with the multiparticle-fields model based on QCD [66].

In addition, the idea of the proposed method allowed us to analyze the energy dependence of the shape of inclusive rapidity distribution and to propose the physical explanation for this effect.

\section{ACKNOWLEDGMENTS}

This work is supported by the Ministry of Education and Science of Ukraine (Project No. 0119U103982). The authors thank the referee, who provided useful comments on the manuscript which improved its clarity. 
[1] D. Amati, A. Stanghellini, and S. Fubini, Theory of highenergy scattering and multiple production, Nuovo Cimento 26, 896 (1962).

[2] E. A. Kuraev, L. N. Lipatov, and V. S. Fadin, The Pomeranchuk singularity in nonabelian gauge theories, Zh. Eksp. Teor. Fiz. 72, 377 (1977) [Sov. Phys. JETP 45, 199 (1977)], http://www.jetp.ac.ru/cgi-bin/e/index/e/45/2/p199?a=list.

[3] M. Baker and K. A. Ter-Martirosyan, Gribov's Reggeon calculus: Its physical basis and implications, Phys. Rep. 28, 1 (1976).

[4] L. N. Lipatov, Bjorken and Regge asymptotics of scattering amplitudes in QCD and in supersymmetric gauge models, Phys. Usp. 51, 631 (2008).

[5] V. S. Fadin, E. A. Kuraev, and L. N. Lipatov, Multiregge processes in the Yang-Mills theory, JETP 44, 443 (1976), http://www.jetp.ac.ru/cgi-bin/e/index/e/44/3/p443?a=list.

[6] M. G. Kozlov, A. V. Reznichenko, and V. S. Fadin, Quantum chromodynamics at high energy, Vestnik NGU, Physics, 2, 3 (2007), http://www.phys.nsu.ru/vestnik/catalogue/ 2007/04/Vestnik_NSU_07T2V4_p3-31.pdf.

[7] C. Duhr and Z. Liu, Multi-regge kinematics and the scattering equations, J. High Energy Phys. 01 (2019) 146.

[8] V.S. Fadin, E. A. Kuraev, and L. N. Lipatov, On the pomeranchuk singularity in asymptotically free theories, Phys. Lett. B 60, 50 (1975).

[9] I. Sharph, A. Tykhonov, G. Sokhrannyi, M. Deliyergiyev, N. Podolyan et al., On the role of longitudinal momenta in high energy Hadron-Hadron scattering, Central Eur. J. Phys. 10, 858 (2012).

[10] I. V. Sharf and V. D. Rusov, Mechanisms of proton-proton inelastic cross-section growth in multi-peripheral model within the framework of perturbation theory. Part 1, J. Mod. Phys. 2, 1480 (2011).

[11] I. V. Sharf, A. J. Haj Farajallah Dabbagh, A. V. Tikhonov, and V. D. Rusov, Mechanisms of proton-proton inelastic crosssection growth in multi-peripheral model within the framework of perturbation theory. Part 2, J. Mod. Phys. 3, 16 (2012).

[12] I. V. Sharf, A. V. Tykhonov, G. O. Sokhrannyi, K. V. Yatkin, and V.D. Rusov, Mechanisms of proton-proton inelastic cross-section growth in multi-peripheral model within the framework of perturbation theory. Part 3, J. Mod. Phys. 3, 129 (2012).

[13] I. V. Sharf, A. V. Tykhonov, G. O. Sokhrannyi, K. V. Yatkin, M. A. Deliyergiyev, N. A. Podolyan, and V. D. Rusov, Description of hadron inelastic scattering by the Laplace method and new mechanisms of cross-section growth, Ukr. J. Phys. 56, 1151 (2011), http://www.jetp.ac.ru/cgi-bin/e/ index/e/44/3/p443?a=list.

[14] I. Sharf, A. Tykhonov, M. Delyiergyiev, G. Sokhrannyi, and N. Podolyan et al., On equivalence of gluon-loop exchange in the inelastic processes in perturbative QCD to pion exchange in $\phi^{3}$ theory, EPJ Web Conf. 60, 20018 (2013).

[15] N. G. de Bruijn, Asymptotic Methods in Analysis MCap (Dover, New York, 2010).

[16] D. R. Snider and D. M. Tow, Inclusion of interference terms in the Amati-Bertocchi-Fubini-Stanghellini-Tonin multiperipheral model, Phys. Rev. D 3, 996 (1971).
[17] D. M. Tow, Some predictions of the Amati-BertocchiFubini-Stanghellini-Tonin multiperipheral model, Phys. Rev. D 2, 154 (1970).

[18] S. Ostapchenko, Monte Carlo treatment of hadronic interactions in enhanced Pomeron scheme: QGSJET-II model, Phys. Rev. D 83, 014018 (2011).

[19] K. Werner, The hadronic interaction model EPOS, Nucl. Phys. B, Proc. Suppl. 175-176, 81 (2008).

[20] G. P. Vacca, J. Bartels, and M. Salvadore, AGK cutting rules and multiple scattering in hadronic collisions, Eur. Phys. J. C 42, 53 (2005).

[21] H. J. Drescher, M. Hladik, S. Ostapchenko, T. Pierog, and K. Werner, Parton-based Gribov-Regge theory, Phys. Rep. 350, 93 (2001).

[22] G. Goldhaber, W. B. Fowler, S. Goldhaber, and T. F. Hoang, Pion-Pion Correlations in Antiproton Annihilation Events, Phys. Rev. Lett. 3, 181 (1959).

[23] V. Khachatryan et al., First Measurement of Bose-Einstein Correlations in Proton-Proton Collisions at $\sqrt{s}=0.9$ and $2.36 \mathrm{TeV}$ at the lhc, Phys. Rev. Lett. 105, 032001 (2010).

[24] CMS Collaboration, Bose-Einstein correlations of charged hadrons in proton-proton collisions at $\sqrt{s}=13 \mathrm{TeV}$, J. High Energy Phys. 03 (2020) 014.

[25] B. Lörstad, Boson interferometry: A review of high energy data and its interpretation, Int. J. Mod. Phys. A 04, 2861 (1989).

[26] G. Alexander, Bose einstein and fermi dirac interferometry in particle physics, Rep. Prog. Phys. 66, 481 (2003).

[27] V. A. Schegelsky, Levy analysis of bose-einstein correlation in pp collisions at $\sqrt{s}=7 \mathrm{TeV}$ measured with the atlas, Phys. Part. Nucl. Lett. 16, 503 (2019).

[28] V. A. Khoze, A. D. Martin, M. G. Ryskin, and V. A. Schegelsky, Two scales in Bose-Einstein correlations, Eur. Phys. J. C 76, 193 (2016).

[29] T. Csörgõ and S. Hegyi, Model independent shape analysis of correlations in 1, 2 or 3 dimensions, Phys. Lett. B 489, 15 (2000).

[30] G. Goldhaber, S. Goldhaber, W.-Y. Lee, and A. Pais, Influence of Bose-Einstein statistics on the anti-proton proton annihilation process, Phys. Rev. 120, 300 (1960).

[31] M. Gyulassy, S. K. Kauffmann, and L. W. Wilson, Pion interferometry of nuclear collisions. I. Theory, Phys. Rev. C 20, 2267 (1979).

[32] W. A. Zajc, Monte carlo calculational methods for the generation of events with Bose-Einstein correlations, Phys. Rev. D 35, 3396 (1987).

[33] R. M. Weiner, Boson interferometry in high-energy physics, Phys. Rep. 327, 249 (2000).

[34] U. A. Wiedemann and U. Heinz, Particle interferometry for relativistic heavy-ion collisions, Phys. Rep. 319, 145 (1999).

[35] G. A. Kozlov, O. V. Utyuzh, and G. Wilk, Bose-Einstein correlation function $C_{2}(q)$ from a quantum field theory point of view, Phys. Rev. C 68, 024901 (2003).

[36] O. Utyuzh, G. Wilk, and Z. Wlodarczyk, Modelling Bose Einstein correlations via elementary emitting cells, Phys. Rev. D 75, 074030 (2007).

[37] G. A. Kozlov, The Bose-Einstein distribution functions and the multiparticle production at high energies, New J. Phys. 4, 23 (2002). 
[38] S. Pratt and V. Zelevinsky, Explaining Centauro Events by Formation of Pions in the Isospin Singlet Channel, Phys. Rev. Lett. 72, 816 (1994).

[39] S. Pratt, Deciphering the centauro puzzle, Phys. Rev. C 50, 469 (1994).

[40] S. Pratt, Pion lasers from high-energy collisions, Phys. Lett. B 301, 159 (1993).

[41] T. Altinoluk, N. Armesto, G. Beuf, A. Kovner, and M. Lublinsky, Hanbury-Brown-Twiss measurements at large rapidity separations, or can we measure the proton radius in p-a collisions, Phys. Lett. B 752, 113 (2016).

[42] E. Gotsman, E. Levin, and U. Maor, Bose-Einstein correlations and $v_{2 n}$ and $v_{2 n-1}$ in hadron and nucleus collisions, Phys. Rev. D 95, 034005 (2017).

[43] E. C. G. Sudarshan and J.R. Klauder, Fundamentals of Quantum Optics (Dover, New York, 2006).

[44] D. J. Scalapino and R. L. Sugar, A statistical theory of particle production, Phys. Rev. D 8, 2284 (1973).

[45] J. C. Botke, D. J. Scalapino, and R. L. Sugar, Coherent states and particle production, Phys. Rev. D 9, 813 (1974).

[46] G. N. Fowler, N. Stelte, and R. M. Weiner, Condensates and Bose-Einstein correlations, Nucl. Phys. A319, 349 (1979).

[47] A. Vourdas and R. M. Weiner, Multiplicity distributions and Bose-Einstein correlations in high-energy multiparticle production in the presence of squeezed coherent states, Phys. Rev. D 38, 2209 (1988).

[48] E. L. Berger, B. Y. Oh, and G. A. Smith, Two-Particle Correlations in Inclusive $\mathrm{p}$ p Interactions between 13 and 28 GeV/c, Phys. Rev. Lett. 29, 675 (1972).

[49] A. I. Golokhvastov, Scaling of semi-inclusive spectra of $\pi^{-}$ mesons from pp interactions, Z. Phys. C 26, 469 (1984).

[50] W. Thome et al., Charged particle multiplicity distributions in p p collisions at ISR energies, Nucl. Phys. B129, 365 (1977).

[51] A. Breakstone et al., Comparison of inclusive distributions in $\mathrm{pp}$ and $\mathrm{pp}$ interactions at $\sqrt{s}=53 \mathrm{GeV}$, Phys. Lett. 132B, 458 (1983).

[52] G. Giacomelli, Inclusive and semi-inclusive hadron interactions at ISR and collider energies, Int. J. Mod. Phys. A 05, 223 (1990).

[53] G. J. Alner et al., Scaling of Pseudorapidity distributions at c.m. energies up to 0.9-TeV, Z. Phys. C 33, 1 (1986).

[54] M. Tanabashi et al. (Particle Data Group), Review of particle physics, Phys. Rev. D 98, 030001 (2018).

[55] A. B. Kaidalov and K. A. Ter-Martirosian, Multiple production of Hadrons at high-energies in the model of quarkGluon strings, Yad. Fiz. 39, 1545 (1984) [Sov. J. Nucl. Phys. 39, 979 (1984)], https://inis.iaea.org/search/search.aspx? orig_q=RN:15032104.
[56] A. B. Kaidalov and K. A. Ter-Martirosyan, Multihadron production at high energies in the model of quark gluon strings, Yad. Fiz. 40, 211 (1984) [Sov. J. Nucl. Phys. 40, 135 (1984)], https://inis.iaea.org/search/search.aspx?orig_q= RN:16073385.

[57] G. Wilk, Fluctuations, correlations and non-extensivity, Braz. J. Phys. 37, 714 (2007).

[58] F. S. Navarra, O. V. Utyuzh, G. Wilk, and Z. Włodarczyk, Information theory in high-energy physics (extensive and nonextensive approach), Physica (Amsterdam) 344A, 568 (2004).

[59] G. Wolschin, Pseudorapidity distributions of produced charged hadrons in pp collisions at RHIC and LHC energies, Europahys. Lett. 95, 61001 (2011).

[60] K. Werner, I. Karpenko, T. Pierog, M. Bleicher, and K. Mikhailov, Evidence for hydrodynamic evolution in protonproton scattering at $900 \mathrm{GeV}$, Phys. Rev. C 83, 044915 (2011).

[61] A. K. Likhoded, A. V. Luchinsky, and A. A. Novoselov, Inclusive light hadron production in $p p$ scattering at the LHC, Phys. Rev. D 82, 114006 (2010).

[62] A. K. Dash and B. Mohanty, Extrapolation of multiplicity distribution in $p+p(\bar{p})$ collisions to LHC energies, J. Phys. G 37, 025102 (2010).

[63] K. Werner, I. Karpenko, T. Pierog, M. Bleicher, and K. Mikhailov, Evidence for hydrodynamic evolution in protonproton scattering at $900 \mathrm{GeV}$, Phys. Rev. C 83, 044915 (2011).

[64] Z.-J. Jiang, H.-Li Zhang, K. Ma, and J. Wang, A description of pseudorapidity distributions in p-p collisions at center-ofmass energy from 23.6 to $900 \mathrm{GeV}$, Chin. Phys. C 39, 044102 (2015).

[65] I. V. Sharf, K. K. Merkotan, N. A. Podolyan, D. A. Ptashynskyy, A. V. Tykhonov et al., Gluon loops in the inelastic processes in QCD, arXiv:1210.3490.

[66] Y. V. Volkotrub et al., Multi-particle field operators in quantum field theory, Phys. J. 2, 181 (2016), https://arxiv .org/abs/1510.01937.

[67] D. Ptashynskiy, T. Zelentsova, N. Chudak, K. Merkotan, O. Potiienko, V. Voitenko, O. Berezovskiy, V. Opyatyuk, O. Zharova, T. Yushkevich, I. Sharph, and V. Rusov, Multiparticle fields on the subset of simultaneity, Ukr. J. Phys. 64, 732 (2019).

[68] K. A. Olive et al., Review of particle physics, Chin. Phys. C 38, 090001 (2014).

[69] NA49 Collaboration, Inclusive production of charged pions in $p+p$ collisions at $158 \mathrm{GeV} / \mathrm{c}$ beam momentum, Eur. Phys. J. C 45, 343 (2006). 